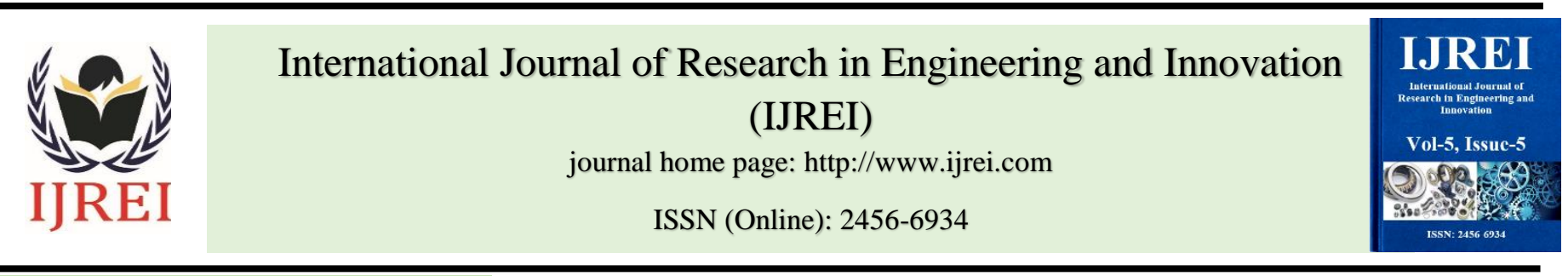

REVIEW ARTICLE

\title{
Application of green chemistry: a review
}

\author{
Omar Salih Hassan', Amina Mohsin Abass², Ahmed Ahmed ${ }^{3}$ \\ ${ }^{I}$ Department of Chemistry, College of Education for Pure Science, Tikrit University, Tikrit, Iraq. \\ ${ }^{2,3}$ Department of Chemistry, College of Science, Al-Nahrain University, Al-Jaderia, Baghdad-Iraq
}

\begin{abstract}
Article Information
Received: 08 June 2021

Revised: 29 June 2021

Accepted: 05 July 2021

Available online: 09 July 2021
\end{abstract}

Keywords:

PVC;

Plasticizers;

Green Chemistry

\begin{abstract}
Green chemicals are as well identified as harmless chemicals. It is use up to project chemical product and procedure which reduce the production of hazardous chemicals. Green chemistry diagonally extends the life cycle of a chemical like: creating, using constructing and eventually disposing of it. Green chemistry is of great help in preventing molecular waste. This offers creative research approaches, this eliminates the negative influence of substances on the environment and human health, even though quiet advancing from the greater assets of plasticized PVC is to exchange the plasticizers which has little molecular weight with harmonious and harmless polymeric plasticizers. The 12 basics of green chemistry (Preventing pollution, Maximizing the atomic energy, model less risky synthesis of chemical agents, make material and goods healthier, using better condition for solvents and reaction and increase energy efficiency etc.). Though the usage of plastic PVC is an imperative role of healthcare worldwide. Novel laws towards the usage of phthalate plasticizers in medical tools, children products and toys is requiring new alternatives to be created. One way to avoid the negative effect of plasticizers migration, although still enjoying greater assets of plasticized PVC, interchange little molecular weight plasticizers by non-toxic ones and friendly polymeric plasticizers.

C2021 ijrei.com. All rights reserved
\end{abstract}

\section{Introduction}

Plasticizers are additives that decrease its viscosity and increase the plasticity of a substance. Applied the chemicals for variation their physical appearance. Either those have low ability solids or liquids to prepare them extra stable, they decrease the attraction amid chains of polymer. Other than 30,000 various compounds have been tested for their plastizing properties over the last 60 years, Just a lesser number of these around 50 are in marketable usage today for plastic, especially poly vinyl chloride(PVC), the dominant applications are when collective with plasticizers, with and related products, clays and concrete, the characterizations of further materials might as well be artificial metric tons[1], in Europe, including1.3 million metric tons, plastic plasticizers are additives, naturally in PVC applications of phthalate esters . Near $90 \%$ of plasticizers are used in PVC, successful durability and strength of this material some are used in cables and movies [2]. It was generally supposed that function of plasticizers by separating them (increasing the "safe volume"), embedding themselves among polymer chains [3] or swell up them and decrease the transition of glass temperature for substantially of plastic and become it easier, Nonetheless, it was later show up that the principle of free volume could not reward for each the effect 
of plasticization [4,5]. Plastic products containing plasticizers can show greater flexibility and durability, due to plastic migration and abrasion, plasticizers may become available for exposure as they are not attached to the matrix of polymer. The "is repeatedly related to products of plasticizers for deprivation or its plasticizers [6,7], nonetheless, because of their exceedingly low vapor pressure and volatility and vapor, multiple studies of smell makeup do not consider phthalate in appreciable quantities. Plasticizers allow for improved processing characteristics of chemicals, while also offering versatility in the finale -use of production. The selection of ester plasticizers is depended on fee- performing evaluations. The compounder of rubber should determine compatibility with plasticizers of ester, efficiency other reliability features. In processing, the wide variety of ester chemicals includes, terephthalates, adipates, sebacates, gluterates di benzoates, azelates, phthalates and additional specialty variations. A wide range of products offers a range of operation values for the several elastomer uses, things for example tubes and pipes, wall covering, walls, ties, seals and gaskets, cable and wire, and printing rolls. In a varied limit of elastomers, together with polychloroprene, nitrile, high to low polarization ester, chlorinated polyethylene EPDM, and epichlorohydrin. The interaction between plasticizers and elastomers is controlled by several influences, for example chemical structure, molecular weight, compatibility solubility parameter and efficiency characteristics are fundamental factors for a particular application in creating a rubber formulation $[8,9]$.

\subsection{PVC: Poly (vinyl chloride)}

PVC is a formless, leaky thermoplastic, simply combined by several additives for obtaining a wide range of material characterizations. Its broad range of mechanical, chemical and physical properties, cost-effectiveness and durability. Consider PVC beneficial for ranging of applications from containers to healthcare equipment, toys, construction materials, electrical wire insulation, clothing or furnishing. Shown the usage of PVC in Fig. 1. To boost the PVC properties, a compatible plasticizer is introduced to changes its thermal characterization and mechanical $[9,10]$ This is achieved by splitting the molecules of PVC to the contact between polymer chains segments. That results in lower adhesion between molecules [11-14]. Additionally, the application of plasticizer to PVC decreases either the flexibility function and the point of glass transition. The results in an increase in PVC manufacturing characterizations, some plasticizers have not chemically joined to PVC chains of polymer [11,14]. The study of coverage to these composites was therefore an important part of preserving health of public and minimizing negative consequences [15]. The traditional view of plasticizers is that to create a workable plasticizer, we must strike a balance between the reliability, performance and durability of the PVC-mixed plasticizer. The fact that beneficial results are achieved with regard to of products, certain properties may have an adverse effect molecular characteristics like polar group on a plasticizer for example attract locations on the ring of PVC and make the plasticizer less PVC-compatible, but if there are just components that polar in a plasticizer, is not very high in plasticizing efficacy. Alternatively, non -polar molecule segments typically have strong plasticization, but if the plasticizer is too big or too multiple, it could be poorly mix with PVC which lead to bloodshed, this meticulous balancing act to maximize plasticizer output the research and development sector has been in the forefront for many years. In the present years, it has made an attempt to develop novel environment friendly plasticizers(TPE)based on vegetable oil fatty acid. The chemical structure of synthesized plasticizers is similar to vegetable oil but with higher degree of branching than vegetable oil. Chemical structure of the obtained plasticizer was characterized with FT-IR and 1H NMR. The thermal stability, plasticizing performance, solvent resistance of the plasticized PVC blends with TPE were investigated and compared with DOP [16].

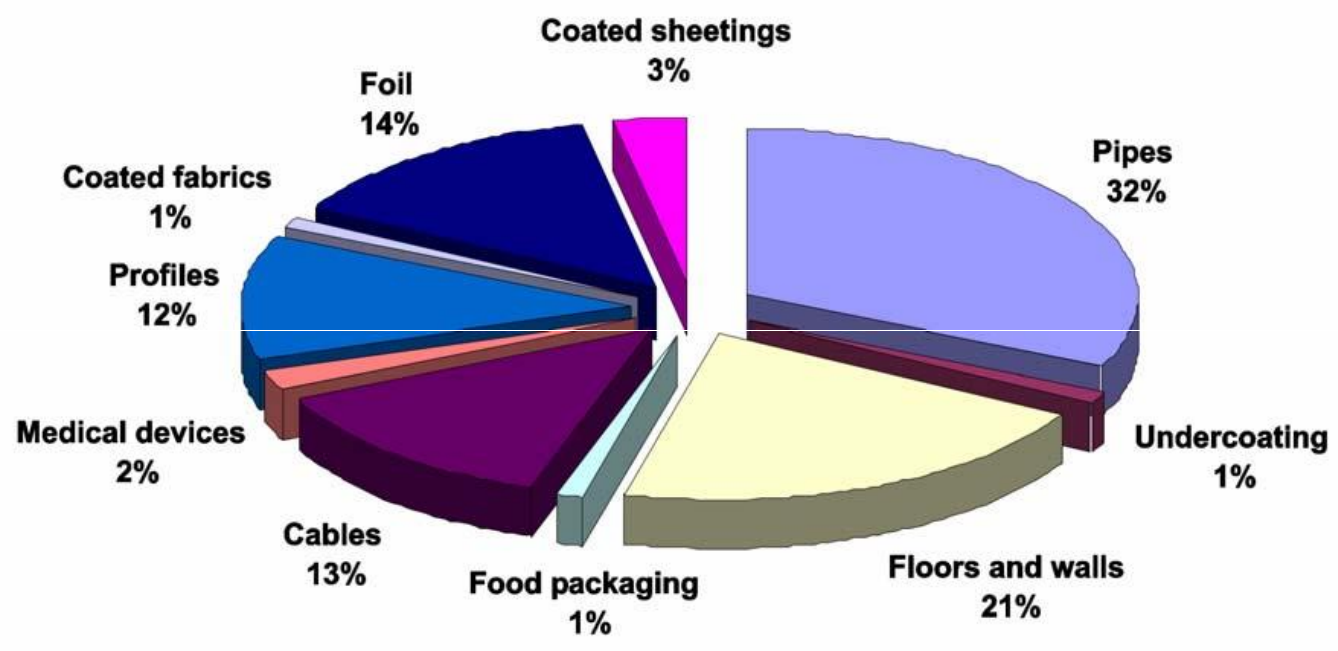

Figure 1: Usage of PVC areas [1] 


\subsection{Preparation of plasticized PVC [12]}

A series of plasticized PVC blends were prepared with the formulations shown in Table 1. Firstly, PVC powder, plasticizers and thermal stabilizers (the mass ratio: $\mathrm{CaSt} 2 / \mathrm{ZnSt} 2=2 / 1$ ) were mixed using a mechanical mixer at room temperature for $10 \mathrm{~min}$. Second, the mixture was compounded at $160^{\circ} \mathrm{C}$ for 5 min by using a double-roll mill Then, the films were made using a hot press at $180^{\circ} \mathrm{C}$ for 2 $\mathrm{min}$. The blends were cooled to room temperature to obtain soft PVC samples.

Fourier transform infrared (FTIR) analysis was conducted using a FALA2000104 spectrometer by an attenuated total reflectance method. The specimens were scanned from 4000 $\mathrm{cm}^{-1}-500 \mathrm{~cm}^{-1}$.

${ }^{1} \mathrm{H}$ NMR spectrum of the compounds in deuterated chloroform $\left(\mathrm{CDCl}_{3}\right)$ was recorded with a Bruker $400 \mathrm{MHz}$ spectrometer.

TOF-MS was recorded with a MALDI spectrometer.

Thermogravimetric analysis (TGA) was carried out using an

1100SF TG instrument. The plasticizers were scanned from $50^{\circ} \mathrm{C}$ to $400^{\circ} \mathrm{C}$ and the PVC samples to $600^{\circ} \mathrm{C}$ under a nitrogen atmosphere at a heating rate of $20^{\circ} \mathrm{C} / \mathrm{min}$. Differential scanning calorimetry (DSC) of the PVC samples was performed from $-20^{\circ} \mathrm{C}$ to $200^{\circ} \mathrm{C}\left(10^{\circ} \mathrm{C} / \mathrm{min}\right)$ using a MettlerDSC822e instrument. Mechanical properties were measured according to ISO 5275:1997 on an electronic tensile testing machine with a gage length of $10 \mathrm{~mm}$. The extension rate was $50 \mathrm{~mm} / \mathrm{min}$, and five specimens were used for each PVC sample to obtain an average value. Shore A durometer hardness was determined in accordance with ISO 868:2003. Five measures at $15 \mathrm{~s}$ per sample were performed.

Table 1: Formulation of the PVC films [12]

\begin{tabular}{|l|l|l|l|l|l|}
\hline Component & F0 & F1 & F2 & F3 & F4 \\
\hline PVC $(\mathrm{g})$ & 100 & 100 & 100 & 100 & 100 \\
\hline ALCH $(\mathrm{g})$ & 00 & 15 & 30 & 45 & 60 \\
\hline ATBS $(\mathrm{g})$ & 60 & 45 & 30 & 15 & 00 \\
\hline Thermal stabilizers $(\mathrm{g})$ & 3 & 3 & 3 & 3 & 3 \\
\hline
\end{tabular}

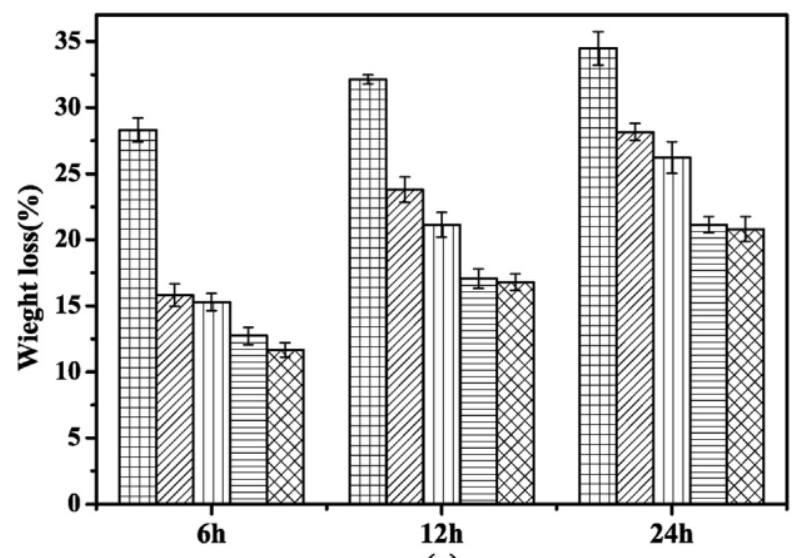

(a)

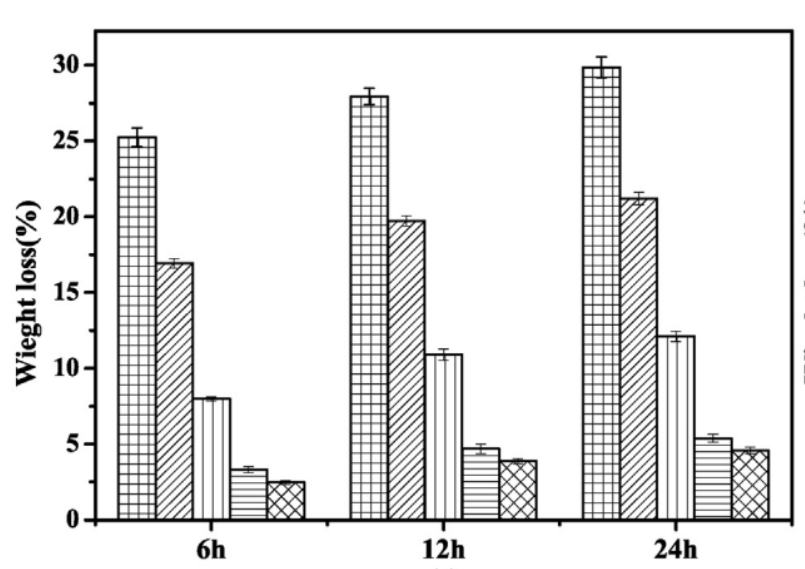

(c)

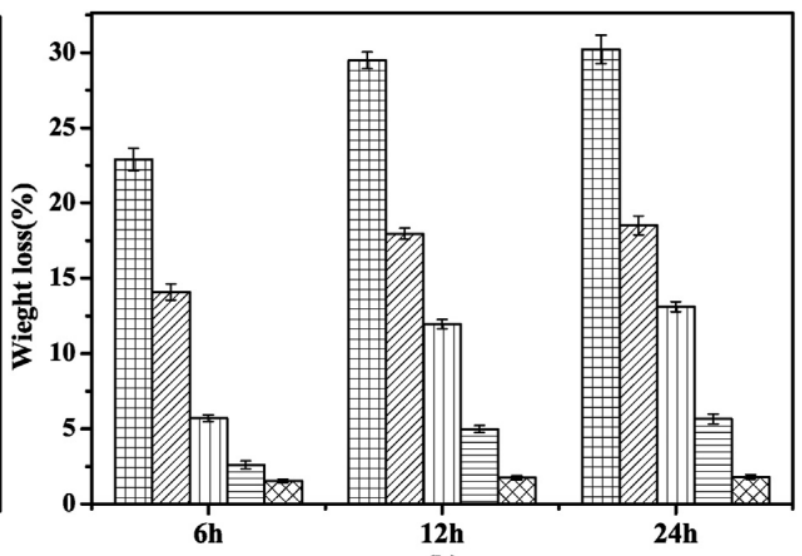

(b)

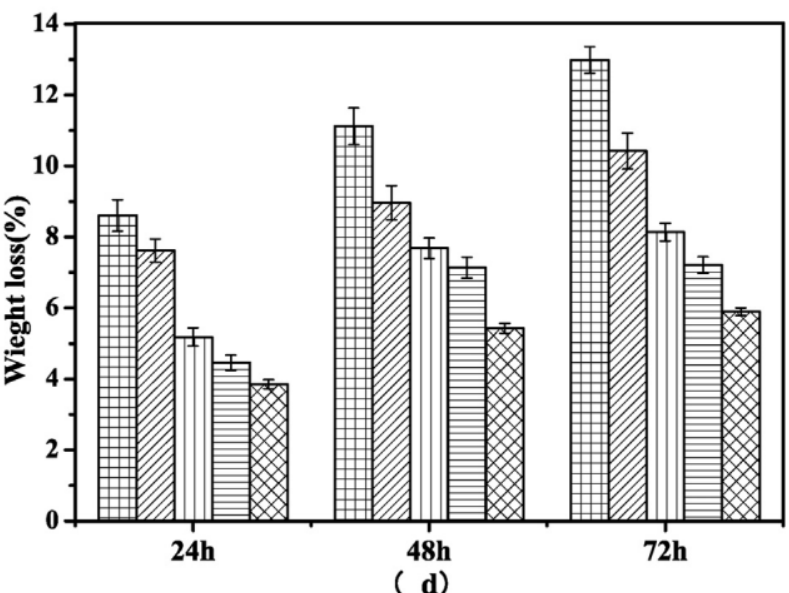

(d)

F0, שIFF1, ШF2, $\boxminus F 3$,

(a) Anhydrous ethanol, (b) Cyclohexane, (c) Petroleum ether, (d) Activated carbon Figure 2: Weight losses of PVC films after migration and exudation testing [12] 
Migration and volatility resistance of PVC films was explored and shown in Fig. 2. The polarity of the molecules, the molar mass and volume, the plasticization process and the physical properties of the plasticizer may affect migration and volatility stability. In addition, the surrounding temperature, the contact area with other media and other conditions also play important roles [17]. It can be clearly observed that the weight loss of different PVC films followed the order of F0 $>$ F1 $>$ F2 $>$ F3 $>$ F4. With the substitution of 4-cyclohexanedimethyl ester (ALCH) into acetyl tributyl citrate (ATBC), the migration and volatility stability was improved, especially in weakly polar solvents such as cyclohexane and petroleum ether (The mass loss rates were reduced by $94.08 \%$ and $84.66 \%$, respectively). This result can be explained by that a large number polar groups on ALCH interact with the polar parts of the PVC chains, which means better compatibility between ALCH and PVC matrix. As a result, ALCH could give PVC films longterm stability and reduce the impact on contact materials. In addition, migration continued with time up to $24 \mathrm{~h}$, which is different from a previous study [18].

\subsection{Types of plasticizer}

Plasticizers can be divided into two major groups: primary and secondary. Primary plasticizers lower the glass transition temperature $\mathrm{T}$ and increase the elongation and softness of the polymer. Secondary plasticizers, when added to the polymer alone, do not bring about such changes and may also have limited compatibility with the polymer. However, when added to the polymer in the presence of a primary plasticizer, secondary plasticizers enhance the plasticizing performance of the primary plasticizer.

\subsubsection{Use of plasticizer}

The list of commercially available plasticizers, covering applications throughout polymer science, is extensive. This reflects the development of plasticizers to meet the increasing demand for polymers, especially PVC, for new and higher quality applications. This article discusses the main groups of plasticizers; for further details, see $[19,20]$ and references therein. The Western European plasticizer market had a capacity of $969 \times 10^{3}$ t/a in 1989 and at that time was growing at a rate of $3 \%$ per annum [21]. In 1996 production of plasticizer in Western Europe amounted to $1253 \times 10^{3}$ and in the United states to $636 \times 10^{3}$ t/a [22]. In terms of plasticizer types, the majority of this tonnage $(>85 \%)$ is standard phthalate (esters of phthalic anhydride with $\mathrm{C}_{8}-\mathrm{C}_{10}$ alcohols). Reasons for this are the relatively low price and ready availability of feedstock.

\section{Green Chemistry Basics [23, 24]}

\subsection{Preventive steps}

Preventive waste is better than processing or purifying waste after it has been produced

\subsection{Economy of Atoms}

Synthetic processes should be designed to maximize the integration into the final product of all materials used during the process.

\subsection{Middling Chemical Synthesis}

Synthetic approaches should be used whenever possible Conceived for the use and production of substances with little or no risk to human health and environment.

\subsection{Safer Chemicals Model}

Products of chemical should be engineered to affect the role they seek while reducing their toxicity.

\subsection{Safer auxiliaries and solvents}

Wherever practicable, the use of support substances (e.g., solvent (e.g., separation agents, solvents, etc.) should be prepared needless and harmless while use up.

\subsection{Technology of Efficiency for Energy}

Chemical processes require energy, its economic impacts and environmental should be understood and minimized, synthetic processes should be carried out at ambient pressure and temperature where possible.

\subsection{Usage of stockpiles of Renewable Feed}

Renewable raw material or feedstock should be used instead of reducing whenever technologically and economically feasible.

\subsection{Reduce yields}

Unnecessary derivatives (using blocking groups), protection/defense, temporary chemical / physical process modification), when appropriate, should be minimized or stopped because these steps require additional reagents and could, produce waste.

\subsection{Catalyst}

Catalytic reactive are equivalent to stoichiometric reagents (as selective as possible).

\subsection{Right for deterioration}

Products of chemical should be fabricated so that at the end of their function they break down into innocuous degradation products and do not persist in the environment. 


\subsection{Real-time Pollution Control Research}

Analytical methodologies need to be established further to allow real-time use in process monitoring and management of hazardous substances prior to their development.

\subsection{Safer Chemistry to Avoid Accidents}

Materials and the type of a material used in Chemical methods should be selected to eliminate chemical incident potentials, Including launches, flames, and explosions.

\section{Green Chemical in Application}

\subsection{Glucose Chemicals}

There are the chemical compounds which represent a collection of chemicals which could be made to meet international markets on an absolutely massive scale, glucose is the replacement for chemicals from goods. The production of fragrant compounds is regulated using biotechnological strategies], compounds like catechol, hydroquinone and adipic acid may be synthetic in any substance that may be essential to them, through modifying benzene amid glucose, benzene is the main substance used for these materials will help to lower the use of diverse toxic reagents [25].

\subsection{Polymeric polysaccharides}

They are an important compound group which includes package widely distributed, the wide range of compounds can be harnessed polysaccharide, because the feedstock must be used as starting materials because it is much more environmentally friendly feedstock, these are renewable and benefit from renewability or viability in lieu of feedstock to petroleum. On the other hand, these have no residual risk to the environment and human health. Another few responses:

\subsubsection{Chemical Green Reactions}

Production of aromatic amines which are halide free, the modern development of aromatic is achieved by use the nitrogen to treat benzene with chlorine. Then displace chlorine with a brand new (nucleophilic substitution) group,in this process, nitrobenzene is heated together with aniline to formtetramethyl-ammonium salts in the presence of tetramethyl ammonium hydroxides, the method prevents the use of intermediate halogenations. Homogenous catalysis and an economic system for nuclear power. Trost advanced the system.: The goal of this work is to reduce the pointlessness of products of atoms produced during the process [25].

\subsubsection{Re actives of green}

Reactor for oxidation of Liquids: This allows organic chemicals to oxidise safely by pureoxygen, this may cause reactions at low temperatures and is pretty helpful. as a result, the volume of vent gas was reduced.

\subsubsection{Complexes created by oxidizing green}

Different methods of oxidation have poor environment impacts. The contaminant (metal ion) can be reduced by the use of molecular oxygen as oxidant number one. Most ligands have been developed in oxidizing conditions, which are fast against oxidative decomposition, this makes the synthesis of stable excessive oxidation kingdom transition complexes viable for miles.

\subsubsection{Synthesis of the non-phosgenic isocyanate}

The main critical polymers are the polyurethane, usually, polyurethanes are prepared with phosgene, phosgene has however the downside of being highly toxic fuel, the acute reason for giving up is lethality. Atechnique is used where precursors of poly urethanes and their isocyanate are synthesized to keep away from the poisonous gas phosgene). For solvents that are high in quantity and acceptable, the potential for its negative effects on human health and the environment can be extremely high, diverse solvents are convenient and difficult to handle [26].

\subsubsection{Drug processing}

Drugs that are oligonucleotides: Drugs used in artificial oligonucleotides expand the number of drug molecules with a wide array of healing properties. Apparently, HL-30TM (a polystyrene bead) is used at a dosage of $90 \mathrm{mmol} / \mathrm{g}$. It exhibits various limiting properties:

- Not biologically degradable

- Non-regenerative

- This about contributes about 40 per cent of the fees for raw materials.

- It is a raw material of a single supply.

\subsubsection{Green chemicals products}

Certain ways to build nitrites: Similar compound structures th at could be toxic are studied and improvements to decrease toxicity it is thought that the process that causes acute toxicity is due to the removal of hydrogen cyanide from cyanohydrins, depending on the existence of the alpha carbon substitution feature it can be decreased or strengthened.

\section{Polygenic aspartic acids (donlar's)}

Soya cyst nematode fungus is still a problem for agriculture. Specific glycinoeclepins are part of an interdisciplinary effort to find a bio logical solution to the problem. An analog (a natural nematode hatching stimulus) were developed and tested. Amount of analogs found to have inhibitory effect on 
soybean cyst nematode eggs hatching such eggs will last in the

\section{Conclusions}

Examples of recent green chemicals product developments, the active developments of environmentally compliant and efficient polymer products have been demonstrated in the co text of sustainable development. The synthesis of different vegetable raw material to form new products will also present a challenge for future research and development, as will the production of additives for polymer or composite applications. Use of Modified Green Components is a convenient way to achieve the goal of green chemistry and is highly recommended for use in polymer areas. To attain further development in this sector, improved process and alteration resulting in improved properties and reduced hazard, should be used instead of petrochemical-based material.

\section{References}

[1] Malveda M.P., " Chemical Economics Handbook Report on Plasticizers,2015 "(https://www.ihs.com/prod ucts/plasticizerschemical-economics-handbook.htm.1).

[2] Tullo A.H.,"Plasticizer Makers Want A Piece Of The Phthalates Pie. In", Chemical \& Engineering News 93(25), 2015, p.p. 16-18.

[3] Cadogan D.F., Howick C.J., "Plasticizers" in Ullmann's Encyclopedia of Industrial Chemistry 2000, Wiley-VCH, Weinheim. doi:10.1002/14356007.a20_439.

[4] Market Study Plasticizers, 3rd ed., Ceresana, Nov. 2013.

[5] Maeda, Y.; Paul, D. R. J." Effect of antiplasticization on gas sorption and transport. I. Polysulfone", Polym. Sci. Part B Polym. Phys. 1987, 25, 957-980.

[6] Maeda, Y.; Paul, D. R. J.," Effect of antiplasticization on gas sorption and transport. II. Poly(phenylene oxide", Polym. Sci. Part B Polym. Phys. 1987, 25, 1005- 1016.

[7] Casalini, R.; Ngai, K. L.; Robertson, C. G.; Roland, C. M. J.," $\alpha$ - and $\beta-$ Relaxations in Neat and Antiplasticized Polybutadiene",Polym. Sci. Part B Polym. Phys. 2000, 38, 1841-1847.

[8] Geiss, O.; Tirendi, S.; Barrero-Moreno, J.; Kotzias, D., "Investigation of volatile organic compounds and phthalates present in the cabin air of used private cars", Environment International 2009, 35, 1188-1195. doi:10.1016/j.envint.2009.07.016

[9] Brydson J.A.,"Plastics materials", 4th Edition, Butterworth Scientific, soil women for 11 to 12 days [27]. London, Chap. 5, 73-85, 1982.

[10] Unar, I.N.; Soomro U.I.N., S.A. and Aziz, S. ,"Effect of various additives on the physical properties of polyvinylchloride resin". Pak. J. Anal. Environ. Chem. Vol. 11, No. 2 (2010) 44 . 50.

[11] Shah,B.L. and Shertukde, V.V.," Effect of plasticizers on mechanical, electrical, permanence, and thermal properties of poly",(vinyl chloride). J. Appl. Polym. Sci. 90, 3278-3284, 2003.

[12] Liu, T.; Jiang, P.; Liu, H.; Li, M.; Dong, Y.; Wang, R. and Wang, Y.," Performance testing of a green plasticizer based on lactic acid for PVC". J. Polym. Test., vol. 61, pp. 205-213, 2017.

[13] Edmund, H. ,Herman M., "Principles of Plasticization", Advances in Chemistry, Vol. 48, Chap. 1,1- 26, 1965.

[14] Shashoua, Y.R.," Inhibiting the Deterioration of Plasticized Poly Vinyl Chloride" A Museum Perspective", Ph.D. Thesis, Technical University of Denmark, Lyngby, Denmark, 2001.

[15] Kamrin, M.," Phthalate Risks, Phthalate Regulation, and Public Health": a Review. J. of Toxicology and Environmental Health, Part B, 12, 157174, 2009.

[16] Tong H., Jinping H. J.," Synthesis of a novel environmental friendly plasticizer based on tung oil fatty acid for poly (vinyl chloride) blends", P2o0li1s8h Journal of Chemical Technology, 20, 2, 92-97, 10.2478/pjct2018-0028.

[17] A.P. Tüzüm Demir, S. Ulutan, Migration of phthalate and non-phthalate plasticizers out of plasticized PVC films into air, J. Appl. Polym. Sci. (2012) 1948-1961.

[18] L. Bernard, R. Cueff, C. Breysse, B. Decaudin, V. Sautou, G. Armed Study, Migrability of PVC plasticizers from medical devices into a simulant of infused solutions, Int. J. Pharm. 485 (2015) 341-347.

[19] J. K. Sears, J. R. Darby: The Technology of Plasticizers, Wiley and Sons, New York 1982.

[20] D. L. Buszard in W. V. Titow (ed.): PVC Technology, 4th ed., Elsevier, Amsterdam 1984.

[21] M. Kampmann, Kunststoffe 79 (1989) no. 10, 897.

[22] K. Weissermel, H.-J. Arpe, Industrielle Organische Chemie, WileyVCH, Weinheim, Germany 1998, p. 428.

[23] Boyer, R.F. ,"Effect of plasticizers on some physical properties of polymers",. TAPPI J. 1951, 34, 357-362.

[24] Anastas, P.T.;Warner, J.C.," Green Chemistry: Theory and Practice"; Oxford University Press: Oxford, UK, 1998.

[25] Wardencki W, Curyo J, Namieoenik J.,"green chemistry- current and future issues", Polish Journal of Environmental Studies Vol. 14, No 4 (2005), 389-395.

[26] Sheldon R.A., " Metrics of Green Chemistry and Sustainability: Past, Present, and Future", ACS Sustainable Chem. Eng. 2018, 6, 1, 32-48.

[27] Iwata T., Miki H., Fujita Y. ,"Ullmann's Encyclopedia of Industrial Chemistry", Vol. A19,VCH, Weinheim, 1991, p. 347.

Cite this article as: Omar Salih Hassan, Amina M. Abass, Ahmed Ahmed, Application of green chemistry: a review, International journal of research in engineering and innovation (IJREI), vol 5, issue 5 (2021), 263-268.

https://doi.org/10.36037/IJREI.2021.5507 